Review

\title{
Exosomes in Nasopharyngeal Carcinoma
}

Yujuan Zhou ${ }^{1 \#, ~ L o n g z h e n g ~ X i a ~}{ }^{1 \#, ~ J i n g g u a n ~ L i n ~}{ }^{1}$, Heran Wang ${ }^{1}$, Linda Oyang ${ }^{1}$, Shiming Tan ${ }^{1}$, Yutong Tian ${ }^{1}$, Min $\mathrm{Su}^{1}$, Hui Wang1, Deliang Cao ${ }^{1,2}{ }^{凶}$, Qianjin Liao ${ }^{1 凶}$

1. Hunan Key Laboratory of Translational Radiation Oncology, Hunan Cancer Hospital and The Affiliated Cancer Hospital of Xiangya School of Medicine, Central South University, 283 Tongzipo Road, Changsha 410013, Hunan, China.

2. Department of Medical Microbiology, Immunology \& Cell Biology, Simmons Cancer Institute, Southern Illinois University School of Medicine. 913 N. Rutledge Street, Springfield, IL 62794, USA.

\# Authors contributed equally.

$\square$ Corresponding author: Qianjin Liao or Deliang Cao, Hunan Key Laboratory of Translational Radiation Oncology, Hunan Cancer Hospital and The Affiliated Cancer Hospital of Xiangya School of Medicine, Central South University, 283 Tongzipo Road, Changsha 410013, Hunan, China. Tel: 86-731-88651681; Fax: 86-731-88651999; Email: march-on@126.com or dcao@siumed.edu.

(C) Ivyspring International Publisher. This is an open access article distributed under the terms of the Creative Commons Attribution (CC BY-NC) license (https://creativecommons.org/licenses/by-nc/4.0/). See http://ivyspring.com/terms for full terms and conditions.

Received: 2017.08.24; Accepted: 2017.11.29; Published: 2018.02.11

\begin{abstract}
Exosomes are nanosized (30-100nm) membrane microvesicles secreted through a complex cellular process. Exosomes contain a variety of bioactive molecules, such as proteins, microRNAs(miRNAs or miRs) and long non-coding RNAs (IncRNAs), playing an important role in the cell-to-cell substance transportation and signal transduction. Nasopharyngeal carcinoma-related exosomes (NPC-Exo) have been identified in circulating blood and contribute to tumor cell proliferation, angiopoiesis, and immune tolerance through remodeling of tumor microenvironment (TME). Nasopharyngeal carcinoma-related exosomes may also induce epithelial-mesenchymal transition (EMT), thus promoting tumor metastasis and chemoradioresistance. Clinically, the exosomes may serve as novel biomarkers for diagnosis and targeted therapies of nasopharyngeal carcinoma. This review article updates the understanding of exosomes in nasopharyngeal carcinoma(NPC).
\end{abstract}

Key words: Nasopharyngeal carcinoma; exosomes; chemoradioresistance; tumor microenvironment; miRNA; non-coding RNA

\section{Introduction}

Nasopharyngeal carcinoma (NPC) is a malignant epithelial tumor associated with Epstein-Barr (EB) virus infection[1]. NPC is endemic with a high prevalence in Southern China, Asia and North Africa, but its incidence is increasing yearly worldwide, being a serious public health issue[2]. The exact etiology of NPC remains unclear, but risky factors recognized include EB-virus infection, genetic predispositions, dietary habits and environmental factors $[3,4]$. Exosomes emerge as a critical player in the development and progression of NPC.

Exosomes are 30-100nm double membrane microvesicles originated from cell membrane. Through budding of the endosomal membrane, vesicle-laden endosomes are produced, referred to as multivesicular endosomes (MVEs) or multivesicular bodies (MVBs)[5, 6]. The MVEs fuse with payloads from endoplasmic reticulum, forming exosomes[6] (Figure 1). MVEs may also fuse with lysosomes and enter the degradation pathway[7]. Exosomes from different sources hold different components, but in general they contain proteins, lipids, nucleic acids and other substances[8-10]. To date, 4,563 proteins, 194 species of lipids, 1,639 mRNAs, and 764 miRNAs and other non-coding RNAs(ncRNAs), as well as mtDNA, ssDNA and dsDNA, are identified from exosomes[11-13]. Secreted from cells, exosomes are present in intercellular space and body fluids, such as blood, saliva, urine and cerebrospinal fluid[14, 15]. Through membrane fusion with targeted cells, the exosomal components enter recipient cells and participate in numerous important physiological and pathological processes[16-21]. Some proteins are shared by all exosomes, such as CD9, CD63, CD81 and 


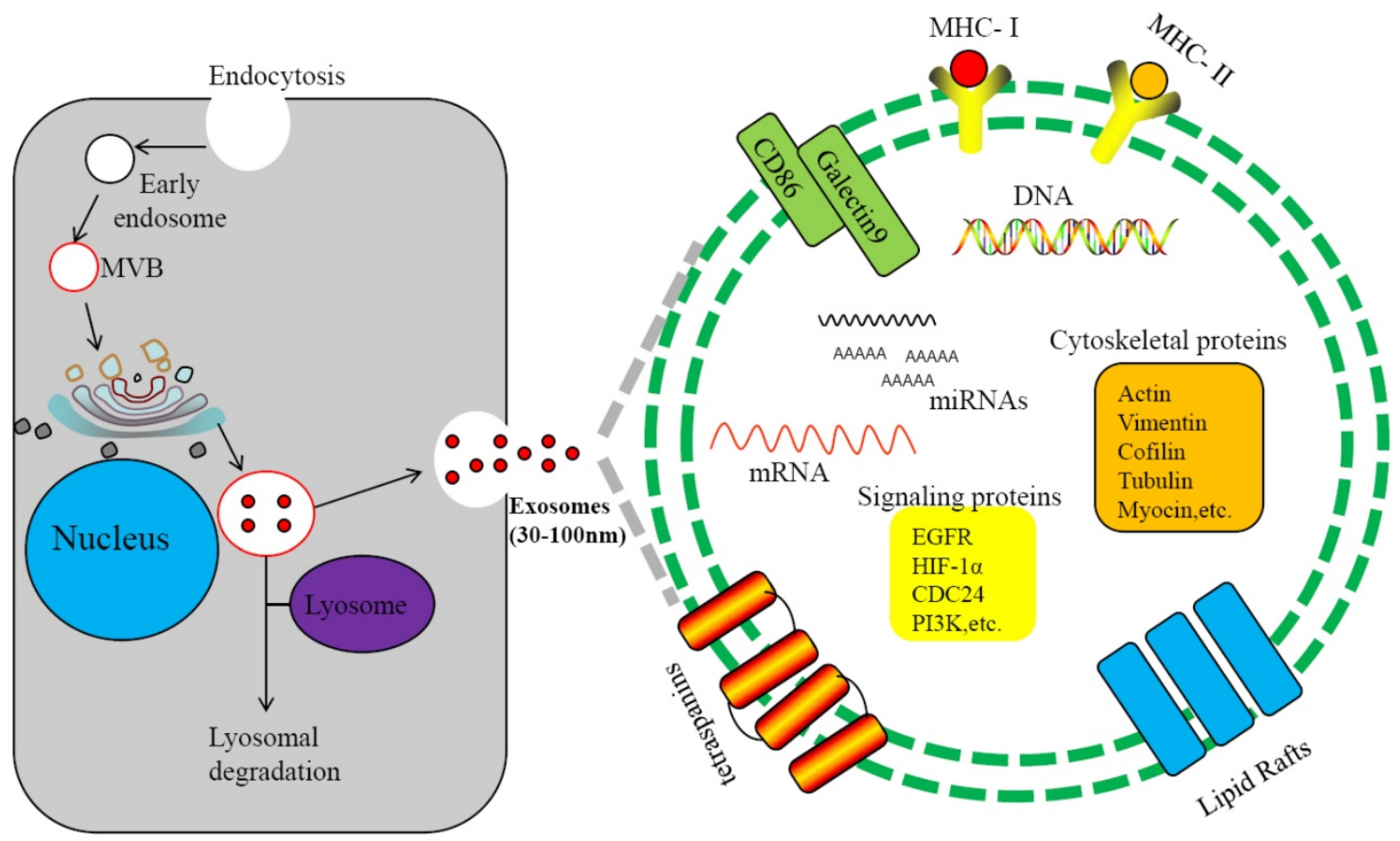

Figure 1. Biogenesis and components of exosomes. Invagination of the plasma membrane form early endosomes, followed by budding of payload into the endosomal membrane to form multivesicular endosomes(MVB). Maturation of the late endosome through acidification triggers fusion with the plasma membrane and release of exosomes.Some of the late endosome fuse with lysosomes for lysosomal degradation. The exosome contains various cellular ingredients, such as lipid, RNAs (including mRNA, miRNA, and non-coding RNA), DNAand proteins, which act as key molecules of signaling transduction and antigen presentation.

CD82[22-24], but exosomes also contain tissue-specific proteins. For example, human nasopharyngeal carcinoma-derived exosome (NPC-Exo) may contain LMP1, an EB viral protein. Therefore, exosomes may function as intercellular vehicles for substance transfer and may also serve as biomarkers for disease diagnosis[25-28].

In cancer, exosomes may participate in exchanges of genetic information between tumor cells and stromal cells and promote angiogenesis and cancer cell growth, migration, invasion and distant metastasis[29-32]. For instance, the exosomes produced by EB virus-infected NPC cells contain LMP1 and viral microRNAs that activate signaling pathways in recipient cells, such as Wnt, PI3K/Akt, JNK, MAPK and NF-KB[33-36]. The EB-virus primary oncogene LMP1 in exosomes can stimulates secretion of pro-invasive fibroblast growth factor 2, promoting NPC cells invasion[37]. Exosomes are also involved in latent infection of viruses[12, 38], and the tumor viruses, such as EB-virus and Kaposi Sarcomaassociated Herpes Virus (KSHV), may manipulate tumor microenvironment through secretion of exosomes containing specific viral components[39].

\section{Nasopharyngeal Carcinoma and Exosomes}

Different kinds of exosomes have been isolated from the serum of NPC patients, such as EBV-related exosomes and pure nasopharyngeal carcinomaderived exosomes[40]. These exosomes contain different contents and thus play differential roles in tumor-host crosstalk and modulate the immune response, angiogenesis, cell proliferation, cell-to-cell communication, and tumor invasion[41, 42]. For instance, the miRNAs derived from the nasopharyngeal carcinoma-derived exosomes regulate expression of genes involved in cell proliferation, differentiation, and stress response[43]. Actually, the nasopharyngeal carcinoma-related exosomes not only include EBVrelated exosomes[44], nasopharyngeal carcinomaderived exosomes[40], mesenchymal stem cell (MSC)derived exosomes[45], and human EBV-transformed lymphoblastoid cell line (LCL)-derived exosomes, etc[46]. It can also contain many exosomes derived from dendritic cells and bone marrow progenitor cells, etc[47-50]. Here importantly elucidate the components and functions of EBV-related exosomes and nasopharyngeal carcinoma-derived exosomes and briefly introduce other exosomes.

\subsection{EBV-related exosomes}

EBV, a gamma herpesvirus, is a major human pathogen and the first human tumour virus identified in Burkitt lymphoma, classic Hodgkin lymphoma (HL), nasopharyngeal carcinoma, and gastric cancer, etc[51]. The first exosomes that contained viral proteins were identified from B cells infected with EBV. Exosomes with the context of infected viral 
components are crucial for viral pathogenesis and can induce local immune tolerance, contributing to viral replication and associated pathogenesis[46]. Oppositely, these exosomes could also restrict viral replication by triggering host immune responses[52, 53]. The exosomes derived from the cells infected with EBV, named as EBV-related exosomes, contain EBNA1, LMP-1, LMP-2A, EBERs, and BARTs encoded by EBV[46]. EBV-related exosomes can be secreted from different type of cells, such as EBV-positive NPC cells or EBV-transformed lymphoblastoid cell lines (LCLs). The recent research found that the major EBV oncoprotein, LMP1, was identified in exosomes secreted from tumor cells that were infected with EBV [54, 55]. During the process of EBV infection, the EBV-related exosomes activates integrin, actin, and nuclear factor kappa B (NF-kB) to help the virus to escape from host immune response, producing a favorable microenvironment for replication of viral particles and tumorigenesis[39]. The EB-viral products that are often integrated, including EBV latent membrane protein 2A, EBV nuclear antigen-1 and EBV non-coding RNAs, etc, are detected in the NPC cells infected with EBV. Now briefly introduce the components of EBV-related exosomes(LMP1, EBNA1 and EBV non-coding RNAs).

\section{EB-virus Latent Membrane Protein 1 and 2A}

EB-virus latent membrane protein 1 (LMP1) and 2A (LMP2A) are the most important viral products and the components of EBV infection-related exosomes. LMP1 can inhibit $\mathrm{T}$ cell activation and thereby promotes tumor cells to escape from the host immune surveillance[56]. LMP1 is required for the progression of EBV-infected cells from the latent status to the cleavage status and is also a viral protein with malignant transformation activity of $B$ lymphocytes, mouse fibroblasts and human epithelial cells.

LMP1 can be localized to internal Golgi and multivesicular body (MVB) compartments and packaged into exosomes for release from the cell[57]. The exosomal LMP1 can mediate viral replication and immunosuppression, establish latency, and promote cell growth[37]. This LMP1 is the most important viral product and the component of EBV-related exosomes. In nasopharyngeal carcinoma, blockage of exosomal LMP1 can inactivating the expression of protooncogene Bcl-2 and survivin through the NF-KB and AP-1 signaling pathways, leading to apoptotic resistance of tumor cells[58] and may also activate HIF-1a signaling cascade and thus enhance invasion of nasopharyngeal carcinoma cells[37]. Oppositely, exosomal LMP1 may induce apoptosis by modulating p53 phosphorylation at multiple sites, such as Ser15,
Ser20, Ser392 and Thr81, depending on the cell types[59]. LMP1 or LMP1 activated signaling molecules delivered by exosomes could facilitate the growth of adjacent cells that are not infected by EBV[60]. LMP1 secretion via exosomes may therefore serve to limit injurious downstream signaling by decreasing LMP1 levels within the cell, thus supporting intracellular virus survival.

LMP2A is also found in EBV-related exosomes. LMP2A is an EB-viral protein mediating viral latency and pathogenesis by functionally mimicking signals of B-cell receptor (BCR). LMP2A alone has not promoting activity in nasopharyngeal carcinoma, but through interaction with LMP1, the LMP2A transferred via exosomes may exert profound effects on cell proliferation, migration, anchorage independence and tumorigenicity. For instance, LMP1 and LMP2A together drive p53 degradation and c-myc expression by activating the PI3K/AKT cascade, leading to undifferentiated and highly metastatic nasopharyngeal carcinoma[60]. Cell growth signaling activated by exosomal LMP1 and LMP2A together including NF- $\mathrm{KB}, \mathrm{PI} 3 \mathrm{~K} / \mathrm{Akt}$ and MAPK pathways.

\section{EB-virus Nuclear Antigen-1 (EBNA-1)}

EBNA1 is an essential protein for the maintenance of viral genome and plays an important role in the EB virus latent infection. The exosomal EBNA1 also induces promyelocytic cell division (PML-NB) leading to DNA replication stress and genomic instability. In addition, exosomal EBNA1 can interact with USP7 to prevent the down-regulation of p53[61, 62]. Ectopic expression of EBNA1 in nasopharyngeal carcinoma cell line $\mathrm{CNE}$ leads to decrease in the expression of epithelial markers but increase in mesenchymal cell markers, inducing EMT[63]. Polymorphisms of the EBNA1 may be a risky factor of cancer[64]. For instance, the NPC-variant of EBNA1 showed less efficiency in episome maintenance in NPC cell line SUNE-1 [65], which is mechanistically linked to chromatin structure and epigenetic modifications, stabilizing viral chromosome transmission during mitosis and viral gene expression [66]. In addition, exosomal EBNA1 may regulate the growth of EBV-infected cells synergistically with viral and cellular oncogenes[60]. However, EBNA1-specific T cells can effectively kill EBV-positive tumor cells, and therefore, EBNA1 may be a potential candidate antigen for immunotherapy of NPC.

\section{EBV non-coding RNAs}

EBV non-coding RNAs (ncRNAs) include EBV-encoded small noncoding RNA 1 and 2 (EBER1 and EBER2) and EBV-miRNAs[67]. EBERs are 
expressed in infectious mononucleosis and many EBV-associated diseases[68]. In NPC patients, EBERs are found in exosomes isolated from their serum. Exosomal EBERs can suppress apoptosis and promote cell growth and proliferation through mediating IGF-1 signaling; expression of EBERs in EBV-negative cells promotes cell growth and leads to resistance to apoptosis[67]. EBV-encoded miRNAs were also detected in EBV-related exosomes that were secreted from cells infected with EBV and that these viral miRNAs could then be transferred to uninfected recipient cells[69]. The exosomal miRNAs encoded by EBV include EBV-miR-BHRF-1 and EBV-miR-BHATs (BamHI-A rightward transcripts, BARTs). The exosomal EBV-encoded BHRF1 miRNA cluster are not expressed in NPC, but the exosomal EBV-miRBARTs are present in the serum of mice transplanted with human nasopharyngeal carcinoma and in the serum of nasopharyngeal cancer patients [70]. The miRNAsproduced by EBV-positive nasopharyngeal epithelial cells can be transferred to the EBV negative cells through the exosomes. Apoptotic genes regulated by exosomal miR-BARTs include PUMA, Bim and TOMM22[71-73]. These exosomal EBERs and miRNAs are involved in the regulation of immune response and in development and progression of NPC (Figure 2). The exosomes play a critical role in the transfer and tumorigenic action of EBV miRNAs[74].

\subsection{Nasopharyngeal Carcinoma-Derived Exosomes}

Tumor-derived exosomes are harbingers of tumor-induced immune suppression [75]. Nasopharyngeal carcinoma-derived exosomes (NPC-Exo) carry molecular markers (e.g., CD63), LAMP1, major histocompatibility complex class I (MHC-I) and class II (MHC-II), HSP70 and miRNAs(Figure 3). NPC-Exo are present in the tumor microenvironment [76] and serum of NPC patients [77, 78]. The roles of NPC-Exo in tumor biology are complex. NPC-Exo contains tumor antigens which could be presented to the cytotoxic $\mathrm{T}$ lymphocyte (CTL) by antigen presenting cells (APC) to produce tumor killing effects; NPC-Exo also promotes the conversion of conventional $\mathrm{CD}^{+} / \mathrm{CD} 25^{-} \mathrm{T}$ cells (Tconv) into Treg and enhances Treg chemoattraction via CCL20. The interaction between NPC-Exo and Treg regulates tumor peripheral tolerance, leading to escaping from immune surveillance[79]. NPC-Exo may promote tumor progression and T-cell dysfunction through the enriched exosomal microRNAs, which modulates the MAPK and JAK/STAT pathways[77, 80].

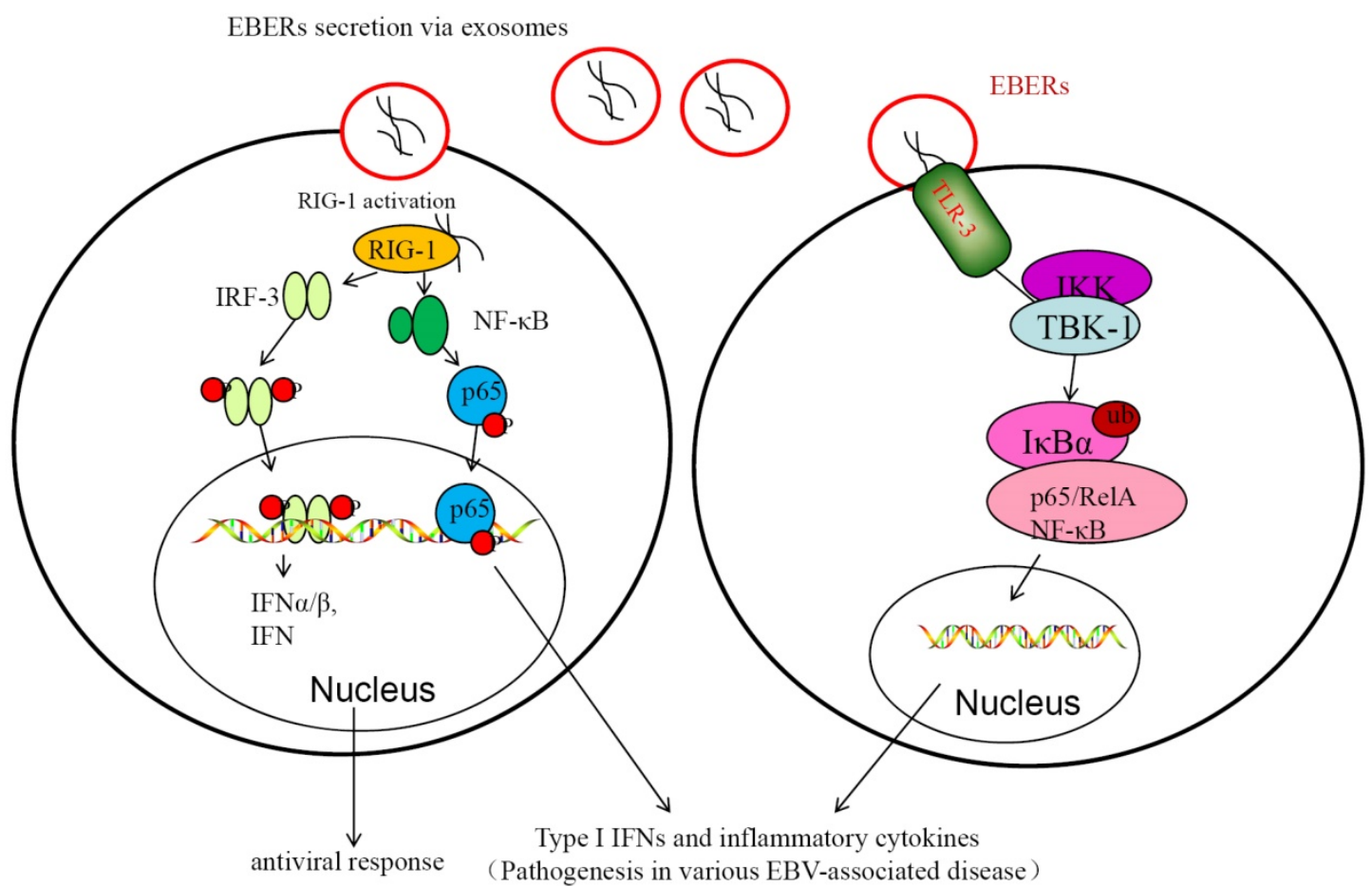

Figure 2. EBER-mediated innate immune response contributes to EBV-mediated pathogenesis. In the left, EBERs transferred through exosomes could be recognized by RIG-I, and then RIG-I is activated in EBV-infected cells. The activated RIG-I triggers IRF3 and NF-KB and stimulates expression of type I IFNs and inflammatory cytokines. This may play important role in maintaining the infectionor antiviral response. In the right, activation of innate immunity via TLR3 signaling in response to secreted EBER. EBER is released from EBV-infected cells through the exosome secretion. EBERs induce type I IFN and inflammatory cytokines via TLR3 signaling cascade, promoting latent infection of EB-virus and occurrence of NPC. 


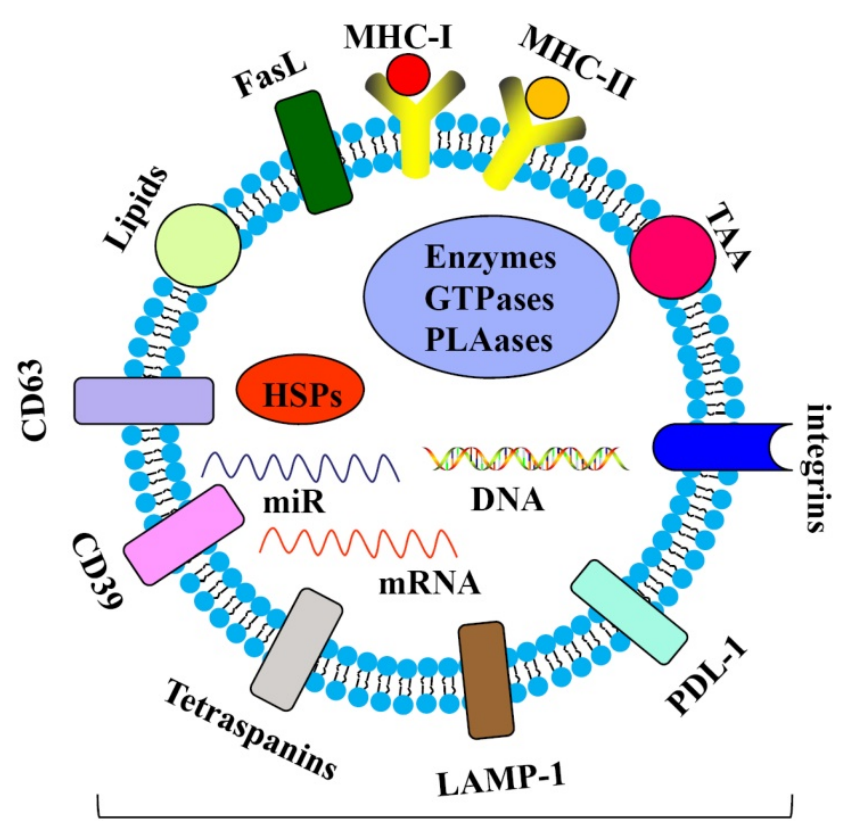

30-120nm

Figure 3. Components of the NPC-derived exosomes. The NPC-derived exosomes may contain immunoinhibitoryand immunostimulatory molecules, such as CD39, PDL-1, LMP-1 and lipids, which are associated with tumor development.

To date, 326 miRNAs derived from NPC have also been detected in NPC-Exo by miRNA chip microarray analysis, and 1511, 1686, and 1229 miRNAs have been identified in the exosomes released from the NPC cell lines $\mathrm{TW}_{03}\left(\mathrm{EBV}^{+}\right)$and TW03 $\left(\mathrm{EBV}^{-}\right)$and the normal NP cell line NP69, respectively[77]. Among them, five miRNAs, miR-24-3p, miR-891a, miR-106a-5p, miR-20a-5p and miR-1908, were commonly present in the exosomes from patient-serum and TW03 $\left(\mathrm{EBV}^{+}\right)$and TW03 $\left(\mathrm{EBV}^{-}\right)$cells[77]. These miRNAs may contribute to intercellular communication and affect the function of tumor cells. For instance, targeted expression of miR-24-3p in NPC-Exo inhibits T-cell proliferation and differentiation by suppressing ERK and STAT signaling pathways[81]. Besides the miR-24-3p, the other four miRNAs can also alter the proliferation and differentiation of T-cell via the phosphorylation of ERK and STAT proteins through the MAPK pathway. Except it, the previous research has noted that hsa-miR-20a-5p targets the JAK1 gene to regulate the JAK/STAT signaling pathway[82]. The recent study found that miR-20a-5p secreted by the radio-resistant NPC cells and transferred to the adjacent NPC cells by the exosomes promoted them radio-resistance via repression of Rab27B by targeting Rab27B 3'-UTR, a newly identified target of miR-20a-5p[83]. Up to now, many miRNAs have been shown to involve in nasopharyngeal carcinoma pathogenesis through alteration of gene networks[84]. However, there's huge room for research about the effect of miRNAs derived from NPC-Exo on NPC.

In addition, NPC-Exo may also promote tumor angiogenesis and cancer cell invasion and metastasis by modulating the tumor microenvironment[43]. The NPC-Exo in serum is positively correlated with lymph node metastasis and shorter survival time, being a potential marker of prognosis.

\subsection{Other Exosomes}

In addition to the EBV infection-derived exosomes and NPC-Exo, other exosomes with distinct sources are isolated from the serum of NPC patients or cell culture, named as NPC relevant exosomes, such as mesenchymal stem cell (MSC)-derived exosomes[85] and human EBV-transformed lymphoblastoid cell line (LCL)-derived exosomes[46]. The exosomes derived from MSCs can modulate the tumor microenvironment and promote cancer cell proliferation and aggressiveness; the nasopharyngeal carcinoma cells co-cultured with MSC-derived exosomes show stronger invasion and metastasis[85]. In fibroblast growth factor (FGF) family, FGF-19 that binds to and activate FGFR4 can be secreted into serum and act in an endocrine fashion. Thus FGF19 expressed in MSC-exosomes could promote proliferation, migration and invasion of NPC cells through activation of the FGF19/FGFR4/ERK signaling cascade[85]. To be noted, MSC-exosomes may inhibit the growth and survival of tumor cells[86]. This may be ascribed to variations inco-culture time of MSCs, exosomes, tumor types, in vivo tumor models, and heterogeneity of MSCs.

Human EBV-transformed lymphoblastoid cell lines (LCLs) are produced by infection of peripheral blood mononuclear cells (PBMC) with EBV. LCLs are stable in genetic materials and easy to maintain in vitro, and thus have been great cell models for investigation of EBV infection in the past decades[87, 88]. The exosomes derived from LCLs are abundant with EBV derivatives, such as ebv-miR-BART3 and ebv-miR-BHRF1-1 and may be a novel model for investigation of EBV infection[46].

\section{Functions of Exosomes in Nasopharyngeal Carcinoma}

Different NPC exosomes may contain diverse contents, but overall, they promote tumor growth and progression through complex mechanisms[77]. Some exosomes may induce cancer resistance to chemoradiotherapy, leading to treatment failure. Thus exosomes are deeply involved in NPC development, progression and prognosis. Here introduce the effect of exosomes on NPC in following aspects. 


\subsection{EBV infection and Immune Surveillance}

EBV infection is closely related to the development and progression of nasopharyngeal carcinoma, but it is still to be clarified how EBV infection promotes tumorigenesis. New evidence indicates that exosomes may play as an important mediator in this EBV-tumorigenic progress. The EBV-encoded products and host functional proteins carried in exosomes can induce EBV escaping from the host immune suppression and thus maintaining infection status[84]. Exosomes secreted by LCLs contained both viral and human cellular miRNA[69], in addition to EBV proteins[33]. The recent study found that all the EB-viral miRNAs expressed in LCL are also packaged in the exosomes[46]. Among these miRNAs, EBV-miR-BART3 and EBV-miR-BHRF1-1, are highly differentially expressed in exosomes derived from LCLs. EBV-mi-RBART3 can induce the pro-inflammatory cytokine IL-6, and be implicated in the regulation of host innate immunity via targeting importin-7 (IPO7), and targeting caspase 3 exerting an anti-apoptotic effect to maintain EBV infection[89, 90]. The BHRF1 miRNA cluster appears to strongly potentiate the transforming properties of EBV, and in particular it has been found that miR-BHRF1-1 potentiates viral lytic replication by down-regulating host p53 in NPC, resulting in the persistent infection of EBV[89]. In addition, the apoptotic resistance induced by exosomal LMP1, together with Th1 cells apoptosis induced by exosomal galactose (galectin-9), promotes NPC cells to escape the immune surveillance[56, 91]. The exosomal dUTPase encoded by EBV induces human dendritic cells, Th- 1 and Th- 17 to secret cytokines and promotes tumorigenesis and inflammation[92], and the EBER in EBV-related exosomes maintains the pool of latent-infected memory B cells during EBV infection[93]. Exosome containing viral miRNAs can influence the surrounding microenvironment, as demonstrated by miR-BART15 that inhibits the NLRP3 inflammasome in surrounding non-infected cells[94]. Similarly, exosomes derived from nasopharyngeal carcinoma cell line TW03 can inhibit the differentiation of Th-17, Th- 1 and cytotoxic T cells, thus weakening the host immune surveillance [79]. In brief, exosomes can mediate $\mathrm{T}$ cell proliferation, differentiation and secretion of cytokines and induce $\mathrm{T}$ cell dysfunction, thereby maintaining EBV latent infection.

\subsection{Intercellular communication and Nasopharyngeal Carcinoma Progression}

Exosomes may function as a powerful way of intercellular transportation of signal molecules[95]. These exosomes, such as NPC-Exo, similarly contain proteins, lipids, and DNA and RNAs. These molecules may function in signaling transduction or contain genetic information to mediate functional interactions between cancer cells and the tumor microenvironment[96]. For instance, the cytokines in exosomes may be released to function as autocrine or paracrine molecules to affect the parental cells or distant target cells. TGF- $\beta$ signaling can be mediated by DNA, miRNA, mRNA, lipids and proteins derived from exosomes in squamous cell carcinoma(SCC), including NPC[97]. The T $\beta R I I$ present in exosomes isolated from the tumor microenvironment can be transferred into NPC cells and stimulate TGF $\beta$ signaling that are unresponsive to TGF $\beta$ ligand in the absence of exosomal transfer in NPC cells. Let-7e, mir-15a, mir-186, mir-26b, mir-224, mir-31, and mir-590 are the most abundant miRNAs transferred by NPC-Exo[40]. They regulate gene expression involved in cancer development, cell proliferation and differentiation, and stress response, playing oncogenic, tumor-suppressive or chemo-resistant roles in NPC $[98,99]$. The exosomes build up a bridge for mutual communication between the host cells and the surrounding tumor cells and/or stromal cells[100, 101], and the increase in exosome release and imbalance of oncogenic and suppressive exosomal miRNAs could have an additional effect on NPC development[40].

The signal molecules that NPC-related exosomes carry may include inhibitory signals which induce immune tolerance and escaping, angiogenin and vascular endothelial growth factor (VEGF) that stimulate angiogenesis, and cytokines and growth factors that promote cell division and tumor growth[102]. Of course, the NPC-Exo may also function in presentation of specific tumor antigens and induce anti-tumor immune response. The double edge sword roles of exosomes in tumor immune response may reflect on stages of nasopharyngeal carcinoma, which would produce exosomes with different protein and nuclei acid contents. NPC-Exo also functions in cancer metastasis. HAX-1 is an anti-apoptotic protein of Bcl-2 family, promoting apoptotic resistance. The HAX-1 protein level in NPC-Exo is closely associated with lymph node metastasis, clinical stages and prognosis of NPC[103]. The C666-1-derived exosomes exhibited a high level of angiogenic proteins, such as ICAM-1 and CD44v5[104], while TSP-1, an angio-suppressive protein, was enriched in non-tumorous NP69 and NP460 exosomes[105]. NPC-Exo-induced migration, invasion and tubulogenesis of NPC cells would possibly be mediated through the uptake of exosomes that contain differential amounts of proangiogenic and angiosuppressive proteins that alter signaling pathways of Src kinase, ERK1/2 kinase, p38 MAPK, 
RhoA/ROCK and endothelial nitric-oxide synthase (eNOS)[106, 107]. In addition, exosomal proteins, exosomal miRNAs are important factors in the invasion and metastasis of NPC. For example, miR-9 is present in human $5-8 \mathrm{~F}$ cell-derived exosomes[78, 108]. Downregulation of miR-9 by promoter methylation leads to upregulation of CXCR4, stimulating proliferation, migration and invasion of NPC cells by activating the MAPK pathway[109]. This miR-9 also regulates the host immune response to NPC by targeting IFN inducible genes, e.g., MHC-I and interleukin genes[110]. In addition, EBV-miRBART-10-3p in EBV infection-derived exosomes can induce EMT of nasopharyngeal carcinoma cells, promoting invasion and metastasis [111]. Apart from facilitating tumor angiogenesis, NPC-Exo can also enter into the circulation and conditioned distal sites for metastasis and maintained the communication between primary and metastatic tumors.

In summary, multiple components in nasopharyngeal carcinoma-related exosomes are involved in cell proliferation, apoptosis, adhesion and invasion. These differentially expressed components in the NPC-related exosomes are involved in the formation, growth and progression of this malignant diseases via targeting different receptors [112].

\subsection{Chemoradioresistance}

The relapse of NPC range from $15 \%$ to $58 \%$ after receiving primary chemoradiotherapy, and the sensitivity to radiotherapy or chemotherapy of relapsed tumorsis decreased. Therefore, the chemoradioresistanceand recurrence of NPC have become the most sophisticated issues of clinicians. The molecular mechanisms of chemoradioresistance are diversified, including transporter pumps, oncogenes, tumor suppressor genes, mitochondrial alterations, DNA repair, autophagy, epithelialmesenchymal transition (EMT) and cancer stemness[113-115]. Exosomes may contribute to drug resistance through transfer into sensitive cancer cells of proteins, miRNAs, and lncRNAs that endorse a resistance phenotype [25]. The exosome contents derived from NPC-associated fibroblast or mesenchymal stem cells may be transferred from donor to recipient cells, and control a wide range of pathways involved in drug resistance by establishing a fertile microenvironment, suppressing immune surveillance and removing chemotherapeutic drugs and radiotherapy[116, 117]. MSC exosomes can induce 5-FU resistance by antagonizing 5-FU-induced apoptosis, activating CaM-Ks/Raf/MEK/ERk pathway, and promoting MDR, MRP and LRP expression[118, 119]. In addition, the EBNA1 present in EBV-related exosomes can inhibit miR-200a and
miR-200b and thus promotes EMT and drug resistance[120]. LMP1 in EBV infection-derived exosomes could trigger the phosphoinositide 3kinase/protein kinase $\mathrm{B}$ (PI3K/AKT) pathway to stimulate the stemness and chemoradioresistance of NPC [121, 122].

Typically, EMT cells acquire resistance to apoptosis, which in turn causes chemoradioresistance[123]. Exosomes modulate EMT through interactions with and deposition of exosomal contents (e.g. DNAs, mRNAs, miRNAs) into the recipient cells $[124,125]$. For example, NPC-Exo mediates EMT of cells through transferring mitotic cytokines (e.g., TGF- $\beta$ ), leading to resistance to chemotherapy [123, 126]. Besides, EBV-BART-miRNAs transferred via exosomes induce EMT through targeting the major tumour suppressor, phosphatase and PTEN[127]. Tthe exosomal EBV-BART1 could increase F-actin level and trigger actin cytoskeleton rearrangement in NPC cells by activating FAK-p130 Cas and Shc-MEKERK1/2 pathways, prompting EMT and drug resistance $[128,129]$.

In addition, NPC-related exosomes may also promote fibroblast growth, resulting in desmoplastic reaction that affects the efficient delivery of anti-cancer drugs and thus results in drug resistance[130]. Dysregulation of miRNAs in CAFs (cancer-associated fibroblasts) and exosomal miRNA transfer between cancer cells and the microenvironment is correlated to chemoresistance[131]. The fibroblast-derived exosomes also induce cancer stem cells that contribute to chemo-resistance[132]. LMP-1 in exosomes may stimulate CD44 expression and lead to radiotherapy resistance of NPC cells through inhibition of the AKT signaling pathway and its downstream factor phospho-FOXO1 in EBV-positive NPC cell line[133, 134]. Although many miRNAs in the NPC-related exosomes have the positive effect on the treatment resistance, the exosomal miR-3188 establishes a negative feedback loop via key signaling pathways mTOR, PI3K/AKT and c-JUN, which suppress cell-cycle signal transition, inhibit cell growth and thus sensitize cells to 5-FU in NPC[41, 135, 136]. The role of exosomes in cancer drug resistance is diversified and further study is warranted.

\subsection{Biomarkers for diagnosis and prognosis}

With the rapid development of liquid biopsy[137], exosomes can be detected in patients' serum, urine, semen, saliva, amniotic fluid, cerebrospinal fluid, bile, ascites, tears, breast milk, and blood[25, 138]. These exosomes are derived from cancer cells and released into circulating blood. Particularly, exosomes are highly stable, standing for 
over $96 \mathrm{~h}$ at 4 degrees. Therefore, circulating NPC-related exosomes can be used in liquid biopsies as noninvasive biomarkers for early detection, diagnosis, and treatment monitoring of NPC patients[139]. In NPC, miRNAs, particularly the EBVBART-miRNAs expressed in NPC-related exosomes, appear abnormal in NPC-Exo and play a role in tumorigenesis[140, 141], thus being potential biomarkers for tumor diagnosis and prognosis[141]. In fact, EBV-DNA level has been recommended as a new factor in the prognosis of NPC[142], because most of the prior researches has confirmed improving the efficiency of exosomal EBV DNA level in the prognosis of patients from high-incidence area of NPC $[143,144]$.

In the progression of NPC, the tumor cells constantly release exosomes into the surrounding environment. In NPC patients, the serum concentration of exosomes is positively related to the metabolism of NPC cells. The higher concentration of exosomes is the more active metabolism and physiological activity, being more malignant. Therefore, the concentration of exosomes in the serum of NPC patient is associated with advanced lymph node metastasis and poor prognosis [81]. In addition, the NPC exosomes with high contents of HLA-II, galectin-9[56, 91] and miR-24-3p[81] are indicators for better prognosis of NPC $[145,146]$.

\section{Conclusion}

As an important component of the tumor microenvironment and a carrier of important signal molecules, exosomes play a critical role in intercellular substance transfer and signal transduction, involved in the development and progression of NPC. EBV-related exosomes integrate EBV-encoded products (proteins and ncRNAs) and functional materials of the host cells and to a certain extent, explain the role of EB-virus in the development and progression of NPC. NPC-Exo contains bioactive host molecules that contribute to growth, metastasis, therapeutic resistance, and recurrence of NPC. These exosomes may be biomarkers of NPC for clinical diagnosis and prognosis due to their tumor origins and stability in serum. However, the overall understanding of exosomes is still limited although impressive progresses in exosomal studies have achieved. The molecular mechanisms involved in the production, sorting and secretion of exosomes remain to be clearly clarified. How to enrich certain populations of exosomes, distinguish the useful exosomes and identify tissue or cell sources of exosomes are also challenges[147]. It is also a fact that not a promising NPC exosomal biomarker, is implemented into clinical practice thus far [148], and the potential use of exosomes in the NPC clinics has not been excavated. We should concentrate on excavating more exosomes and clarifying their functions and clinical significance. Especially the clinical trials need to be taken to investigate their effects in NPC patients. Nevertheless, with the great attention of cancer scientists to exosomes, breakthrough progress in exosomes is expectable, which will eventually contribute to clinical management of NPC.

\section{Acknowledgement}

This work was supported in part by grants from the National Natural Science Foundation of China (81472595, 81402006) and the Research Project of Health and Family Planning Commission of Hunan Province (B20180400, B20180582); Development and Reform Commission of Hunan Province.

\section{Conflicts of Interest}

Authors declare no conflicts of interest.

\section{References}

1. Chua, ML, Wee, JT, Hui, EP, et al. Nasopharyngeal carcinoma. Lancet. 2016; 387(10022):1012-24.

2. Ma, BBY, Hui, EPChan, ATC. Investigational drugs for nasopharyngeal carcinoma. Expert Opin Investig Drugs. 2017; 26(6):677-685

3. Liao, Q, Zeng, Z, Guo, X, et al. LPLUNC1 suppresses IL-6-induced nasopharyngeal carcinoma cell proliferation via inhibiting the Stat 3 activation. Oncogene. 2014; 33(16):2098-109.

4. Lung, ML, Cheung, AK, Ko, JM, et al. The interplay of host genetic factors and Epstein-Barr virus in the development of nasopharyngeal carcinoma. Chin J Cancer. 2014; 33(11):556-68

5. Todorova, D, Simoncini, S, Lacroix, R, et al. Extracellular Vesicles in Angiogenesis. Circ Res. 2017; 120(10):1658-1673.

6. Ibrahim, AMarban, E. Exosomes: Fundamental Biology and Roles in Cardiovascular Physiology. Annu Rev Physiol. 2016; 78:67-83.

7. Mayers, JRAudhya, A. Vesicle formation within endosomes: An ESCRT marks the spot. Commun Integr Biol. 2012; 5(1):50-6.

8. Tkach, MThery, C. Communication by Extracellular Vesicles: Where We Are and Where We Need to Go. Cell. 2016; 164(6):1226-32.

9. Blackwell, RH, Foreman, KEGupta, GN. The Role of Cancer-Derived Exosomes in Tumorigenicity \& Epithelial-to-Mesenchymal Transition. Cancers (Basel). 2017; 9(8).

10. Zhang, X, Pei, Z, Chen, J, et al. Exosomes for Immunoregulation and Therapeutic Intervention in Cancer. J Cancer. 2016; 7(9):1081-7.

11. S, ELA, Mager, I, Breakefield, XO, et al. Extracellular vesicles: biology and emerging therapeutic opportunities. Nat Rev Drug Discov. 2013; 12(5):347-57.

12. De Toro J, Herschlik L, Waldner C, et al. Emerging roles of exosomes in normal and pathological conditions: new insights for diagnosis and therapeutic applications. Front Immunol. 2015; 6:203.

13. Mathivanan, S, Fahner, CJ, Reid, GE, et al. ExoCarta 2012: database of exosomal proteins, RNA and lipids. Nucleic Acids Res. 2012; 40(Database issue):D1241-4.

14. Mittelbrunn, MSanchez-Madrid, F. Intercellular communication: diverse structures for exchange of genetic information. Nat Rev Mol Cell Biol. 2012; 13(5):328-35

15. Vlassov, AV, Magdaleno, S, Setterquist, R, et al. Exosomes: current knowledge of their composition, biological functions, and diagnostic and therapeutic potentials. Biochim Biophys Acta. 2012; 1820(7):940-8.

16. King, HW, Michael, MZGleadle, JM. Hypoxic enhancement of exosome release by breast cancer cells. BMC Cancer. 2012; 12:421

17. Umezu, T, Tadokoro, $\mathrm{H}$, Azuma, $\mathrm{K}$, et al. Exosomal miR-135b shed from hypoxic multiple myeloma cells enhances angiogenesis by targeting factor-inhibiting HIF-1. Blood. 2014; 124(25):3748-57.

18. Tadokoro, H, Umezu, T, Ohyashiki, K, et al. Exosomes derived from hypoxic leukemia cells enhance tube formation in endothelial cells. J Biol Chem. 2013; 288(48):34343-51.

19. Kosaka, N, Iguchi, H, Hagiwara, K, et al. Neutral sphingomyelinase 2 (nSMase2)-dependent exosomal transfer of angiogenic microRNAs regulate cancer cell metastasis. J Biol Chem. 2013; 288(15):10849-59. 
20. Whiteside, TL. Immune modulation of T-cell and NK (natural killer) cell activities by TEXs (tumour-derived exosomes). Biochem Soc Trans. 2013; 41(1):245-51.

21. Valadi, H, Ekstrom, K, Bossios, A, et al. Exosome-mediated transfer of mRNAs and microRNAs is a novel mechanism of genetic exchange between cells. Nat Cell Biol. 2007; 9(6):654-9.

22. Pietrowska, M, Funk, S, Gawin, M, et al. Isolation of Exosomes for the Purpose of Protein Cargo Analysis with the Use of Mass Spectrometry. Methods Mol Biol. 2017; 1654:291-307.

23. Liu, S, Hossinger, A, Gobbels, S, et al. Prions on the run: How extracellular vesicles serve as delivery vehicles for self-templating protein aggregates. Prion. 2017; 11(2):98-112.

24. Tanase, CP, Codrici, E, Popescu, ID, et al. Prostate cancer proteomics: Current trends and future perspectives for biomarker discovery. Oncotarget. 2017; 8(11):18497-18512.

25. Kalluri, R. The biology and function of exosomes in cancer. J Clin Invest. 2016; 126(4):1208-15

26. Jin, $\mathrm{H}, \mathrm{Wu}, \mathrm{YTan}, \mathrm{X}$. The role of pancreatic cancer-derived exosomes in cancer progress and their potential application as biomarkers. Clin Transl Oncol. 2017; 19(8):921-930.

27. Inamdar, S, Nitiyanandan, RRege, K. Emerging applications of exosomes in cancer therapeutics and diagnostics. Bioeng Transl Med. 2017; 2(1):70-80.

28. Soung, YH, Ford, S, Zhang, V, et al. Exosomes in Cancer Diagnostics. Cancers (Basel). 2017; 9(1)

29. Liu, H, Wang, J, Chen, Y, et al. NPC-EXs Alleviate Endothelial Oxidative Stress and Dysfunction through the miR-210 Downstream Nox2 and VEGFR2 Pathways. Oxid Med Cell Longev. 2017; 2017:9397631.

30. Bastos, N, Ruivo, CF, Silva, S, et al. Exosomes in Cancer: Use them or Target them? Semin Cell Dev Biol. 2017.

31. Wang, Z, Chen, JQ, Liu, JL, et al. Exosomes in tumor microenvironment: novel transporters and biomarkers. J Transl Med. 2016; 14(1):297.

32. Shao, $Y$, Shen, $Y$, Chen, $T$, et al. The functions and clinical applications of tumor-derived exosomes. Oncotarget. 2016; 7(37):60736-60751.

33. Meckes, DG, Jr., Shair, KH, Marquitz, AR, et al. Human tumor virus utilizes exosomes for intercellular communication. Proc Natl Acad Sci U S A. 2010; 107(47):20370-5.

34. Morris, MA, Dawson, CWYoung, LS. Role of the Epstein-Barr virus-encoded latent membrane protein-1, LMP1, in the pathogenesis of nasopharyngeal carcinoma. Future Oncol. 2009; 5(6):811-25.

35. Morris, MA, Dawson, CW, Laverick, L, et al. The Epstein-Barr virus encoded LMP1 oncoprotein modulates cell adhesion via regulation of activin A/TGFbeta and beta1 integrin signalling. Sci Rep. 2016; 6:19533.

36. Zhang, J, Jia, L, Lin, W, et al. Epstein-Barr Virus-Encoded Latent Membrane Protein 1 Upregulates Glucose Transporter 1 Transcription via the mTORC1/NF-kappaB Signaling Pathways. J Virol. 2017; 91(6).

37. Aga, M, Bentz, GL, Raffa, S, et al. Exosomal HIF1alpha supports invasive potential of nasopharyngeal carcinoma-associated LMP1-positive exosomes. Oncogene. 2014; 33(37):4613-22.

38. Meckes, DG, Jr.Raab-Traub, N. Microvesicles and viral infection. J Virol. 2011; 85(24):12844-54

39. Meckes, DG, Jr., Gunawardena, HP, Dekroon, RM, et al. Modulation of B-cell exosome proteins by gamma herpesvirus infection. Proc Natl Acad Sci U S A. 2013; 110(31):E2925-33

40. Harmati, M, Tarnai, Z, Decsi, G, et al. Stressors alter intercellular communication and exosome profile of nasopharyngeal carcinoma cells. J Oral Pathol Med. 2017; 46(4):259-266.

41. Becker, A, Thakur, BK, Weiss, JM, et al. Extracellular Vesicles in Cancer: Cell-to-Cell Mediators of Metastasis. Cancer Cell. 2016; 30(6):836-848.

42. D'Asti, E, Chennakrishnaiah, S, Lee, TH, et al. Extracellular Vesicles in Brain Tumor Progression. Cell Mol Neurobiol. 2016; 36(3):383-407.

43. Ye, SB, Zhang, H, Cai, TT, et al. Exosomal miR-24-3p impedes T-cell function by targeting FGF11 and serves as a potential prognostic biomarker for nasopharyngeal carcinoma. J Pathol. 2016; 240(3):329-340.

44. Zuo, L, Yue, W, Du, S, et al. An update: Epstein-Barr virus and immune evasion via microRNA regulation. Virol Sin. 2017; 32(3):175-187.

45. $\mathrm{Li}, \mathrm{H}, \mathrm{Liu}, \mathrm{D}, \mathrm{Li}, \mathrm{C}$, et al. Exosomes secreted from mutant-HIF-1alpha-modified bone-marrow-derived mesenchymal stem cells attenuate early steroid-induced avascular necrosis of femoral head in rabbit. Cell Biol Int. 2017.

46. Gallo, A, Vella, S, Miele, M, et al. Global profiling of viral and cellular non-coding RNAs in Epstein-Barr virus-induced lymphoblastoid cell lines and released exosome cargos. Cancer Lett. 2017; 388:334-343.

47. Wang, H, Wu, S, Huang, S, et al. Follistatin-like protein 1 contributes to dendritic cell and T-lymphocyte activation in nasopharyngeal carcinoma patients by altering nuclear factor kappab and Jun N-terminal kinase expression. Cell Biochem Funct. 2016; 34(8):554-562.

48. Liu, H, Chen, L, Liu, J, et al. Co-delivery of tumor-derived exosomes with alpha-galactosylceramide on dendritic cell-based immunotherapy for glioblastoma. Cancer Lett. 2017.

49. $\mathrm{Lu}, \mathrm{Z}, \mathrm{Zuo}, \mathrm{B}, \mathrm{Jing}, \mathrm{R}$, et al. Dendritic cell-derived exosomes elicit tumor regression in autochthonous hepatocellular carcinoma mouse models. J Hepatol. 2017; 67(4):739-748.

50. Barrera-Ramirez, J, Lavoie, JR, Maganti, HB, et al. Micro-RNA Profiling of Exosomes from Marrow-Derived Mesenchymal Stromal Cells in Patients with
Acute Myeloid Leukemia: Implications in Leukemogenesis. Stem Cell Rev. 2017

51. Gala, R, Gandhi, JS, Gupta, G, et al. Study of association of Epstein-Barr virus in lymphomas by Epstein-Barr virus-encoded RNA in situ hybridization: An Indian perspective from a tertiary care cancer institute. Indian J Pathol Microbiol. 2017; 60(3):341-349.

52. Raab-Traub, NDittmer, DP. Viral effects on the content and function of extracellular vesicles. Nat Rev Microbiol. 2017; 15(9):559-572.

53. Shirvani-Dastgerdi, E, Winer, BY, Celia-Terrassa, T, et al. Selection of the highly replicative and partially multidrug resistant rtS78T HBV polymerase mutation during TDF-ETV combination therapy. J Hepatol. 2017; 67(2):246-254.

54. Khan, GAhmed, W. Isolation and Characterization of Exosomes Released by EBV-Immortalized Cells. Methods Mol Biol. 2017; 1532:147-158.

55. Hoshina, S, Sekizuka, T, Kataoka, M, et al. Profile of Exosomal and Intracellular microRNA in Gamma-Herpesvirus-Infected Lymphoma Cell Lines. PLoS One. 2016; 11(9):e0162574.

56. Keryer-Bibens, C, Pioche-Durieu, C, Villemant, C, et al. Exosomes released by EBV-infected nasopharyngeal carcinoma cells convey the viral latent membrane protein 1 and the immunomodulatory protein galectin 9. BMC Cancer. 2006; 6:283.

57. Flanagan, J, Middeldorp, JSculley, T. Localization of the Epstein-Barr virus protein LMP 1 to exosomes. J Gen Virol. 2003; 84(Pt 7):1871-9.

58. Verweij, FJ, van Eijndhoven, MA, Hopmans, ES, et al. LMP1 association with CD63 in endosomes and secretion via exosomes limits constitutive NF-kappaB activation. EMBO J. 2011; 30(11):2115-29.

59. Tao, Y, Shi, Y, Jia, J, et al. Novel roles and therapeutic targets of Epstein-Barr virus-encoded latent membrane protein 1-induced oncogenesis in nasopharyngeal carcinoma. Expert Rev Mol Med. 2015; $17:$ e15.

60. Raab-Traub, N. Nasopharyngeal Carcinoma: An Evolving Role for the Epstein-Barr Virus. Curr Top Microbiol Immunol. 2015; 390(Pt 1):339-63.

61. Sivachandran, N, Cao, JYFrappier, L. Epstein-Barr virus nuclear antigen 1 Hijacks the host kinase CK2 to disrupt PML nuclear bodies. J Virol. 2010; 84(21):11113-23.

62. Sivachandran, N, Sarkari, FFrappier, L. Epstein-Barr nuclear antigen 1 contributes to nasopharyngeal carcinoma through disruption of PML nuclear bodies. PLoS Pathog. 2008; 4(10):e1000170.

63. Frappier, L. Ebna1. Curr Top Microbiol Immunol. 2015 391:3-34.

64. Wang, Y, Liu, X, Xing, X, et al. Variations of Epstein-Barr virus nuclear antigen 1 gene in gastric carcinomas and nasopharyngeal carcinomas from Northern China. Virus Res. 2010; 147(2):258-64.

65. Dheekollu, J, Malecka, K, Wiedmer, A, et al. Carcinoma-risk variant of EBNA1 deregulates Epstein-Barr Virus episomal latency. Oncotarget. 2017; 8(5):7248-7264

66. Lieberman, PM. Chromatin Structure of Epstein-Barr Virus Latent Episomes. Curr Top Microbiol Immunol. 2015; 390(Pt 1):71-102.

67. Iwakiri, D. Multifunctional non-coding Epstein-Barr virus encoded RNAs (EBERs) contribute to viral pathogenesis. Virus Res. 2016; 212:30-8.

68. Ke, K, Wang, H, Fu, S, et al. Epstein-Barr virus-encoded RNAs as a survival predictor in nasopharyngeal carcinoma. Chin Med J (Engl). 2014; 127(2):294-9.

69. Pegtel, DM, Cosmopoulos, K, Thorley-Lawson, DA, et al. Functional delivery of viral miRNAs via exosomes. Proc Natl Acad Sci U S A. 2010; 107(14):6328-33

70. Gourzones, C, Gelin, A, Bombik, I, et al. Extra-cellular release and blood diffusion of BART viral micro-RNAs produced by EBV-infected nasopharyngeal carcinoma cells. Virol J. 2010; 7:271.

71. Marquitz, AR, Mathur, A, Nam, CS, et al. The Epstein-Barr Virus BART microRNAs target the pro-apoptotic protein Bim. Virology. 2011; 412(2):392-400.

72. Choy, EY, Siu, KL, Kok, KH, et al. An Epstein-Barr virus-encoded microRNA targets PUMA to promote host cell survival. J Exp Med. 2008; 205(11):2551-60.

73. Dolken, L, Malterer, G, Erhard, F, et al. Systematic analysis of viral and cellular microRNA targets in cells latently infected with human gamma-herpesviruses by RISC immunoprecipitation assay. Cell Host Microbe. 2010; 7(4):324-34.

74. Li, Z, Duan, Y, Cheng, $\mathrm{S}$, et al. EBV-encoded RNA via TLR3 induces inflammation in nasopharyngeal carcinoma. Oncotarget. 2015; 6(27):24291-303

75. Whiteside, TL. Exosomes and tumor-mediated immune suppression. J Clin Invest. 2016; 126(4):1216-23

76. Xiang, X, Poliakov, A, Liu, C, et al. Induction of myeloid-derived suppressor cells by tumor exosomes. Int J Cancer. 2009; 124(11):2621-33

77. Ye, SB, Li, ZL, Luo, DH, et al. Tumor-derived exosomes promote tumor progression and $\mathrm{T}$-cell dysfunction through the regulation of enriched exosomal microRNAs in human nasopharyngeal carcinoma. Oncotarget. 2014; 5(14):5439-52.

78. Harmati, M, Tarnai, Z, Decsi, G, et al. Stressors alter intercellular communication and exosome profile of nasopharyngeal carcinoma cells. J Oral Pathol Med. 2016.

79. Mrizak, D, Martin, N, Barjon, $\mathrm{C}$, et al. Effect of nasopharyngeal carcinoma-derived exosomes on human regulatory T cells. J Natl Cancer Inst. 2015; 107(1):363

80. Diamond, J, Chapman, J, Ueberheide, B, et al. Tumor-Derived Exosomes as Modulators of Radiation-Induced Anti-Tumor Immunity. Int J Radiat Oncol Biol Phys. 2016; 96(2S):S53. 
81. Ye, SB, Zhang, H, Cai, TT, et al. Exosomal miR-24-3p impedes T-cell function by targeting FGF11 and serves as a potential prognostic biomarker for nasopharyngeal carcinoma. J Pathol. 2016.

82. Doebele, C, Bonauer, A, Fischer, A, et al. Members of the microRNA-17-92 cluster exhibit a cell-intrinsic antiangiogenic function in endothelial cells. Blood. 2010; 115(23):4944-50.

83. Huang, D, Bian, G, Pan, $\mathrm{Y}$, et al. MiR-20a-5p promotes radio-resistance by targeting Rab27B in nasopharyngeal cancer cells. Cancer Cell Int. 2017; 17:32.

84. Yue, PY, Ha, WY, Lau, CC, et al. MicroRNA profiling study reveals miR-150 in association with metastasis in nasopharyngeal carcinoma. Sci Rep. 2017; 7(1):12012.

85. Shi, S, Zhang, Q, Xia, Y, et al. Mesenchymal stem cell-derived exosomes facilitate nasopharyngeal carcinoma progression. Am J Cancer Res. 2016; 6(2):459-72.

86. Bruno, S, Collino, F, Deregibus, MC, et al. Microvesicles derived from human bone marrow mesenchymal stem cells inhibit tumor growth. Stem Cells Dev. 2013; 22(5):758-71.

87. Kalchschmidt, JS, Bashford-Rogers, R, Paschos, K, et al. Epstein-Barr virus nuclear protein EBNA3C directly induces expression of AID and somatic mutations in B cells. J Exp Med. 2016; 213(6):921-8.

88. Tousizadeh, B, Moghim, S, Chaleshtori, ARS, et al. Application of Epstein-Barr Virus for Optimization of Immortalized B-lymphocyte Production as a Positive Control in Genetic Studies. Adv Biomed Res. 2017; 6:80.

89. He, ML, Luo, MX, Lin, MC, et al. MicroRNAs: potential diagnostic markers and therapeutic targets for EBV-associated nasopharyngeal carcinoma. Biochim Biophys Acta. 2012; 1825(1):1-10.

90. Harold, C, Cox, DRiley, KJ. Epstein-Barr viral microRNAs target caspase 3. Virol J. 2016; 13:145.

91. Klibi, J, Niki, T, Riedel, A, et al. Blood diffusion and Th1-suppressive effects of galectin-9-containing exosomes released by Epstein-Barr virus-infected nasopharyngeal carcinoma cells. Blood. 2009; 113(9):1957-66.

92. Ariza, ME, Rivailler, P, Glaser, R, et al. Epstein-Barr virus encoded dUTPase containing exosomes modulate innate and adaptive immune responses in human dendritic cells and peripheral blood mononuclear cells. PLoS One. 2013; 8(7):e69827.

93. Baglio SR, van Eijndhoven MA, Koppers-Lalic D, et al. Sensing of latent EBV infection through exosomal transfer of 5'pppRNA. Proc Natl Acad Sci U S A. 2016; 113(5):E587-96.

94. Haneklaus, M, Gerlic, M, Kurowska-Stolarska, M, et al. Cutting edge: miR-223 and EBV miR-BART15 regulate the NLRP3 inflammasome and IL-1beta production. J Immunol. 2012; 189(8):3795-9.

95. Yu, ST, Wei, YFZhang, J. Research on relationship between extracelluar vesicles and cancer. Modern Oncology. 2016; 08:1302 -1306.

96. Schoepp, M, Strose, AJHaier, J. Dysregulation of miRNA Expression in Cancer Associated Fibroblasts (CAFs) and Its Consequences on the Tumor Microenvironment. Cancers (Basel). 2017; 9(6).

97. Cancer Genome Atlas N. Comprehensive genomic characterization of head and neck squamous cell carcinomas. Nature. 2015; 517(7536):576-82.

98. Zhu, H, Zhou, Y, Castillo-Gonzalez, C, et al. Bidirectional processing of pri-miRNAs with branched terminal loops by Arabidopsis Dicer-like1. Nat Struct Mol Biol. 2013; 20(9):1106-15.

99. Zhou, Y, Huang, Z, Wu, S, et al. miR-33a is up-regulated in chemoresistant osteosarcoma and promotes osteosarcoma cell resistance to cisplatin by down-regulating TWIST. J Exp Clin Cancer Res. 2014; 33:12.

100. Desrochers, LM, Antonyak, MACerione, RA. Extracellular Vesicles: Satellites of Information Transfer in Cancer and Stem Cell Biology. Dev Cell. 2016; 37(4):301-9.

101. Azmi, AS, Bao, BSarkar, FH. Exosomes in cancer development, metastasis, and drug resistance: a comprehensive review. Cancer Metastasis Rev. 2013; 32(3-4):623-42

102. Liu CH, Wu MH, Li GY. Tumor Cells-derived Exosome on Tumor Malignant Progression and Chemotherapy. Chinese Journal of Biochemistry and Molecular Biology. 2014; 30(6): 526 -532.

103. You, B, Cao, X, Shao, X, et al. Clinical and biological significance of HAX-1 overexpression in nasopharyngeal carcinoma. Oncotarget. 2016; $7(11): 12505-24$

104. Kucharzewska, P, Christianson, HC, Welch, JE, et al. Exosomes reflect the hypoxic status of glioma cells and mediate hypoxia-dependent activation of vascular cells during tumor development. Proc Natl Acad Sci U S A. 2013; 110(18):7312-7

105. Chan, YK, Zhang, H, Liu, P, et al. Proteomic analysis of exosomes from nasopharyngeal carcinoma cell identifies intercellular transfer of angiogenic proteins. Int J Cancer. 2015; 137(8):1830-41.

106. Tilghman, RWHoover, RL. The Src-cortactin pathway is required for clustering of E-selectin and ICAM-1 in endothelial cells. FASEB J. 2002; 16(10):1257-9.

107. Tilghman, RWHoover, RL. E-selectin and ICAM-1 are incorporated into detergent-insoluble membrane domains following clustering in endothelial cells. FEBS Lett. 2002; 525(1-3):83-7.

108. Lu, J, Xu, X, Liu, X, et al. Predictive value of miR-9 as a potential biomarker for nasopharyngeal carcinoma metastasis. Br J Cancer. 2014; 110(2):392-8.

109. Lu, J, Luo, H, Liu, X, et al. miR-9 targets CXCR4 and functions as a potential tumor suppressor in nasopharyngeal carcinoma. Carcinogenesis. 2014; 35(3):554-63
110. Gao, F, Zhao, ZL, Zhao, WT, et al. miR-9 modulates the expression of interferon-regulated genes and $\mathrm{MHC}$ class I molecules in human nasopharyngeal carcinoma cells. Biochem Biophys Res Commun. 2013; 431(3):610-6

111. Yan, Q, Zeng, Z, Gong, Z, et al. EBV-miR-BART10-3p facilitates epithelial-mesenchymal transition and promotes metastasis of nasopharyngeal carcinoma by targeting BTRC. Oncotarget. 2015; 6(39):41766-82.

112. Tan, G, Tang, XTang, F. The role of microRNAs in nasopharyngeal carcinoma. Tumour Biol. 2015; 36(1):69-79.

113. Zeng, AL, Yan, W, Liu, YW, et al. Tumour exosomes from cells harbouring PTPRZ1-MET fusion contribute to a malignant phenotype and temozolomide chemoresistance in glioblastoma. Oncogene. 2017.

114. Brasseur, K, Gevry, NAsselin, E. Chemoresistance and targeted therapies in ovarian and endometrial cancers. Oncotarget. 2017; 8(3):4008-4042.

115. Lu, CShervington, A. Chemoresistance in gliomas. Mol Cell Biochem. 2008; 312(1-2):71-80.

116. Chen, WX, Liu, XM, Lv, MM, et al. Exosomes from drug-resistant breast cancer cells transmit chemoresistance by a horizontal transfer of microRNAs. PLoS One. 2014; 9(4):e95240.

117. Zheng, HC. The molecular mechanisms of chemoresistance in cancers. Oncotarget. 2017; 8(35):59950-59964

118. Chen, FZhang, W. The influence of exosomes derived from tumor cells and stromal cells on tumor drug resistance. Chin J Cancer Biother. 2016; 23(03):432-436.

119. Ji, R, Zhang, B, Zhang, X, et al. Exosomes derived from human mesenchymal stem cells confer drug resistance in gastric cancer. Cell Cycle. 2015; 14(15):2473-83.

120. Wang, L, Tian, WD, Xu, X, et al. Epstein-Barr virus nuclear antigen 1 (EBNA1) protein induction of epithelial-mesenchymal transition in nasopharyngeal carcinoma cells. Cancer. 2014; 120(3):363-72.

121. Yang, GD, Huang, TJ, Peng, LX, et al. Epstein-Barr Virus_Encoded LMP1 upregulates microRNA-21 to promote the resistance of nasopharyngeal carcinoma cells to cisplatin-induced Apoptosis by suppressing PDCD4 and Fas-L. PLoS One. 2013; 8(10):e78355.

122. Yang, CF, Yang, GD, Huang, TJ, et al. EB-virus latent membrane protein 1 potentiates the stemness of nasopharyngeal carcinoma via preferential activation of PI3K/AKT pathway by a positive feedback loop. Oncogene. 2016; 35(26):3419-31.

123. Acloque, H, Thiery, JPNieto, MA. The physiology and pathology of the EMT. Meeting on the epithelial-mesenchymal transition. EMBO Rep. 2008; 9(4):322-6.

124. Henderson, MCAzorsa, DO. The genomic and proteomic content of cancer cell-derived exosomes. Front Oncol. 2012; 2:38.

125. Steinbichler, TB, Dudas, J, Riechelmann, $\mathrm{H}$, et al. The role of exosomes in cancer metastasis. Semin Cancer Biol. 2017; 44:170-181.

126. Andarawewa, KL, Erickson, AC, Chou, WS, et al. Ionizing radiation predisposes nonmalignant human mammary epithelial cells to undergo transforming growth factor beta induced epithelial to mesenchymal transition. Cancer Res. 2007; 67(18):8662-70.

127. Cai, L, Ye, Y, Jiang, Q, et al. Epstein-Barr virus-encoded microRNA BART1 induces tumour metastasis by regulating PTEN-dependent pathways in nasopharyngeal carcinoma. Nat Commun. 2015; 6:7353.

128. Yamada, KMAraki, M. Tumor suppressor PTEN: modulator of cell signaling, growth, migration and apoptosis. J Cell Sci. 2001; 114(Pt 13):2375-82.

129. Chetram, MAHinton, CV. PTEN regulation of ERK1/2 signaling in cancer. J Recept Signal Transduct Res. 2012; 32(4):190-5.

130. Quail, DFJoyce, JA. Microenvironmental regulation of tumor progression and metastasis. Nat Med. 2013; 19(11):1423-37.

131. Santos, JC, Ribeiro, ML, Sarian, LO, et al. Exosomes-mediate microRNAs transfer in breast cancer chemoresistance regulation. Am J Cancer Res. 2016; 6(10):2129-2139.

132. Hu, Y, Yan, C, Mu, L, et al. Fibroblast-Derived Exosomes Contribute to Chemoresistance through Priming Cancer Stem Cells in Colorectal Cancer. PLoS One. 2015; 10(5):e0125625.

133. Yang, CF, Peng, LX, Huang, TJ, et al. Cancer stem-like cell characteristics induced by EB virus-encoded LMP1 contribute to radioresistance in nasopharyngeal carcinoma by suppressing the p53-mediated apoptosis pathway. Cancer Lett. 2014; 344(2):260-71.

134. Mei, YP, Zhou, JM, Wang, Y, et al. Silencing of LMP1 induces cell cycle arrest and enhances chemosensitivity through inhibition of AKT signaling pathway in EBV-positive nasopharyngeal carcinoma cells. Cell Cycle. 2007; 6(11):1379-85.

135. Zhao, M, Luo, R, Liu, Y, et al. miR-3188 regulates nasopharyngeal carcinoma proliferation and chemosensitivity through a FOXO1-modulated positive feedback loop with mTOR-p-PI3K/AKT-c-JUN. Nat Commun. 2016; 7:11309.

136. Melo, SA, Sugimoto, H, O'Connell, JT, et al. Cancer exosomes perform cell-independent microRNA biogenesis and promote tumorigenesis. Cancer Cell. 2014; 26(5):707-21.

137. Alix-Panabieres, CPantel, K. Clinical Applications of Circulating Tumor Cells and Circulating Tumor DNA as Liquid Biopsy. Cancer Discov. 2016; 6(5):479-91.

138. Bardelli, APantel, K. Liquid Biopsies, What We Do Not Know (Yet). Cancer Cell. 2017; 31(2):172-179. 
139. Melo, SA, Luecke, LB, Kahlert, C, et al. Glypican-1 identifies cancer exosomes and detects early pancreatic cancer. Nature. 2015; 523(7559):177-82.

140. Yip, TT, Ngan, RK, Fong, AH, et al. Application of circulating plasma/serum EBV DNA in the clinical management of nasopharyngeal carcinoma. Oral Oncol. 2014; 50(6):527-38

141. Lo, AK, Dawson, CW, Jin, DY, et al. The pathological roles of BART miRNAs in nasopharyngeal carcinoma. J Pathol. 2012; 227(4):392-403.

142. Zhang, J, Shu, C, Song, Y, et al. Epstein-Barr virus DNA level as a novel prognostic factor in nasopharyngeal carcinoma: A meta-analysis. Medicine (Baltimore). 2016; 95(40):e5130.

143. Gurtsevitch, VE, Senyuta, NB, Ignatova, AV, et al. Epstein-Barr virus biomarkers for nasopharyngeal carcinoma in non-endemic regions. J Gen Virol. 2017; 98(8):2118-2127.

144. Sharma, R, Huang, X, Brekken, RA, et al. Detection of phosphatidylserine-positive exosomes for the diagnosis of early-stage malignancies. Br J Cancer. 2017; 117(4):545-552.

145. Sun, D, Zhuang, $X$, Zhang $S$, et al. Exosomes are endogenous nanoparticles that can deliver biological information between cells. Adv Drug Deliv Rev. 2013; 65(3):342-7.

146. Gehrmann, U, Naslund, TI, Hiltbrunner, S, et al. Harnessing the exosome-induced immune response for cancer immunotherapy. Semin Cancer Biol. 2014; 28:58-67.

147. McBride, JD, Rodriguez-Menocal, LBadiavas, EV. Extracellular Vesicles as Biomarkers and Therapeutics in Dermatology: A Focus on Exosomes. J Invest Dermatol. 2017; 137(8):1622-1629.

148. Di Meo A, Bartlett J, Cheng Y, et al. Liquid biopsy: a step forward towards precision medicine in urologic malignancies. Mol Cancer. 2017; 16(1):80. 